\title{
Fatores associados ao sobrepeso em escolares ${ }^{1}$
}

\section{Factors associated with overweight in schoolchildren}

\author{
Lenir Vaz GUIMARÃES² \\ Marilisa Berti de Azevedo BARROS 3 \\ Maria Silvia Amicucci Soares MARTINS² \\ Elisabeth Carmen DUARTE ${ }^{4}$
}

\section{R E S U M O}

\section{Objetivo}

Identificar variáveis associadas ao sobrepeso em escolares de Cuiabá, MT, Brasil.

\section{Métodos}

Foi feito um estudo de caso-controle a partir de um inquérito antropométrico, aplicado em uma amostra aleatória de alunos da primeira série do ensino fundamental, com idades entre 6 e 11 anos. Foram incluídos, como casos, os 158 escolares que apresentaram sobrepeso (índice de massa muscular $\geq P 85$ ) e, como controles, 316 crianças sorteadas entre as que apresentaram índice de massa muscular $<P 85$. Informações socioeconômicas, do domicílio, da família e de atividade física dos escolares foram obtidas por meio de entrevistas. Foram tomadas medidas de peso e altura da criança e dos pais por antropometristas treinados. Os dados foram submetidos à análise de regressão logística múltipla hierarquizada.

\section{Resultados}

O sobrepeso foi maior em escolares com renda familiar per capita $>3$ salários mínimos $(O R=3,75)$, que tinham mães de idade entre 25 e 29 anos $(O R=1,74)$ e com nível mais alto de escolaridade $(O R=1,91)$ e com história de apenas uma união conjugal $(O R=2,53)$; também foi maior nos escolares, de sexo feminino $(O R=2,15)$, que possuíam no máximo um irmão $(O R=1,94)$, brincavam $\leq 10 \mathrm{~h}$ por semana $(O R=2,58)$, tinham mães e pais com índice de massa muscular $\geq 30$ ( $O R=7,27$ e 2,65, respectivamente) e nasceram com peso $>3500 \mathrm{~g}(\mathrm{OR}=2,27)$.

\section{Conclusão}

Os resultados apontam que variáveis de diferentes níveis hierárquicos se associam na configuração de contextos favoráveis ao aumento do sobrepeso em escolares e fornecem subsídios para o desenvolvimento de intervenções que considerem os grupos mais vulneráveis à presença de sobrepeso.

Termos de indexação: antropometria; escolar; fatores de risco; sobrepeso.

1 Artigo elaborado a partir da tese da L.V. GUIMARÃES intutilada "Estado nutricional e fatores associados ao sobrepeso em escolares da área urbana de Cuiabá-MT, Brasil". Programa de Pós-Graduação em Saúde Coletiva, Universidade Estadual de Campinas; 2001. Projeto de Pesquisa financiado pela Fundação de Amparo à Pesquisa de Mato Grosso Processo no 3.5.3.2.158/06.2000 E.Fapemat.

2 Departamento de Saúde Coletiva, Universidade Federal de Mato Grosso. Av. Fernando Correa da Costa, s/n, Coxipó, Campus Universitário, 78060-900, Cuiabá, Mato Grosso, Brasil. Correspondência para/Correspondence to: L.V. GUIMARÃES. E-mail:<lvguima@terra.com.br>.

3 Departamento de Medicina Preventiva e Social, Faculdade de Ciências Médicas, Universidade Estadual de Campinas. Campinas, SP, Brasil.

${ }^{4}$ Departamento de Análise da Situação em Saúde, Secretaria de Vigilância em Saúde, Ministério da Saúde. Brasília, DF, Brasil. 


\section{A B S T R A C T}

\section{Objective}

To identify the variables associated with overweight among schoolchildren in Cuiaba, MT, Brasil.

\section{Methods}

A case-control study was conducted in a sample of schoolchildren aging from 6 to 11 years. The study consisted of 158 overweight schoolchildren (body mass index $\geq 85$ th percentile) and 316 children with body mass index <85th percentile randomly chosen as controls. Interviews were held to obtain information regarding the children's socioeconomic status, household conditions, family characteristics and physical activity levels. The children's and the parents' weight and height were measured according to standardized procedures. Multiple, hierarchical logistic regression analysis was applied to the obtained data.

\section{Results}

The results revealed that overweight was higher among schoolchildren with the following characteristics: per capita family income of more than three minimum wages $(O R=3.75)$, the children's mothers with a high level of education ( $O R=1.91)$, a family history of just one marital union $(O R=2.53)$, maternal age ranging from 25 to 29 years $(O R=1.74)$, a maximum of one sibling $(O R=1.94)$, play time per week $\leq 10$ hours $(O R=2.58)$, mothers and fathers with body mass index $\geq 30(O R=7.27$ and 2.65 , respectively), birth weight $>3,500 \mathrm{~g}(\mathrm{O} R=2.27)$ and female gender (OR=2.15).

\section{Conclusion}

The results pointed out variables, at different hierarchical levels, correlated with overweight among schoolchildren. Interventions should consider the complex pattern of obesity determination and the groups at higher vulnerability.

Indexing terms: anthropometry; schoolchildren; risk factors; overweight.

\section{N T R O D U Ç Ã O}

A prevalência de obesidade em crianças e adolescentes tem crescido na maior parte dos países e se traduzido em um dos mais significativos problemas nutricionais da atualidade ${ }^{1,2}$. A obesidade tem se tornado freqüente mesmo em nações em desenvolvimento, nas quais persistem regiões e grupos sociais submetidos a contextos de fome e desnutrição. Estudos apontam, inclusive, a concomitância, de casos de desnutrição e obesidade na mesma moradia ${ }^{3}$.

No Brasil, verifica-se uma tendência de aumento na prevalência de sobrepeso em crianças e adolescentes, entre 6 e 18 anos, de 4,1\% para 13,9\%, no período entre 1975 e $1997^{2}$.

Pesquisadores têm buscado identificar entre as variáveis econômicas, sociais, ambientais e biológicas os fatores associados à ocorrência da obesidade ${ }^{4,5}$. Os achados sobre a relação entre 0 nível socioeconômico e a obesidade infantil não têm sido consistentes na literatura. Entretanto, uma relação inversa, de redução da obesidade, com o aumento da renda familiar, tem sido mostrada em alguns estudos ${ }^{6,7}$. A influência da escolaridade materna também tem revelado resultados discrepantes ${ }^{4,8}$.

A análise de variáveis relacionadas à família e domicílio possibilita delinear o ambiente em que a criança vive. A variável número de irmãos pode ser analisada como um dos indicadores de atividade física da criança, uma vez que quanto mais irmãos a criança tiver mais ela irá brincar ${ }^{9,10}$. As atividades físicas realizadas pela criança na escola e brincadeiras desenvolvidas no lar contribuem na regulação do peso corporal e, conseqüentemente, atuam na prevenção da obesidade 9 . Em oposição, o tempo que a criança assiste televisão, apresenta-se como variável indicadora da inatividade física e com influência positiva na prevalência da obesidade infantil ${ }^{10}$.

A ocorrência de obesidade segundo a distribuição por sexo e idade tem se diferenciado nas investigações ${ }^{1,11}$. Estudo com escolares 
americanos evidenciou maior risco de obesidade em meninas ${ }^{10}$. A literatura tem apontado a idade pré-escolar, próxima aos seis anos, como um dos períodos críticos no desenvolvimento de maior acúmulo de gordura corporal ${ }^{12}$. A relação entre a obesidade dos pais e obesidade dos filhos é evidenciada nos estudos com associações estatísticas fortes ${ }^{4,13}$. Os resultados sobre a influência do peso ao nascer na obesidade infantil também têm sido controversos ${ }^{4,14}$.

A análise da ocorrência de sobrepeso, em um modelo hierarquizado, possibilita identificar as variáveis que melhor explicam o sobrepeso, nos diferentes níveis, e subsidiar a implementação de medidas de controle do risco de obesidade na população.

Num cenário de mudanças no padrão de problemas nutricionais, com prevalência crescente da obesidade e de suas repercussões na saúde, o presente estudo objetivou identificar os fatores associados ao sobrepeso de escolares em uma capital da Região Centro-Oeste do Brasil.

\section{MÉ T O D O S}

Trata-se de um estudo caso-controle, exploratório, realizado com escolares de ambos os sexo, com idade entre 6 e 11 anos, que freqüentavam a primeira série do ensino fundamental das redes de ensino pública e privada da área urbana de Cuiabá, Mato Grosso.

Para realizar o estudo caso-controle, foi estimado o tamanho de amostra capaz de detectar a razão de chance de 2,5; número de controles por caso de 2:1; fator de exposição com freqüência relativa de 10\%; poder de estudo (1- $\beta$ ) de $80 \%$ e nível de significância de 5\%. A amostra mínima calculada foi de 351 escolares, sendo 234 controles e 117 casos $^{15}$. Acrescentou-se 10\% na amostra obtida para compensar possíveis perdas, atingindo o número de 390 crianças sendo 260 controles e 130 casos. Considerando que seriam necessários 130 casos e que a prevalência de sobrepeso nos escolares em Cuiabá seria de 10\% (com base em estudos prévios) chegou-se à amostra do inquérito antropométrico de 1300 escolares. O tipo de amostragem foi estratificada, proporcional e em cluster, constituída por 707, 445 e 148 escolares das redes municipais, estaduais e particulares, respectivamente.

Para inclusão no estudo caso-controle, foram definidos como casos, escolares com índice de massa corporal $\left[\mathrm{IMC}=\right.$ peso $(\mathrm{kg}) /$ altura $\left.(\mathrm{m})^{2}\right]$ igual ou superior ao percentil $85^{\circ}$ (sobrepeso) e, como controles, crianças com IMC menor que o percentil $85^{\circ}$. Os controles foram selecionados por sorteio aleatório simples. Utilizaram-se critérios propostos por Must et al. ${ }^{16}$ para definição dos escolares com e sem sobrepeso.

As criancas e os pais foram pesados com o mínimo de roupa possível e descalços, em balança eletrônica com capacidade de $150 \mathrm{~kg}$ e precisão de $100 \mathrm{~g}$. A altura foi aferida em posição ereta encostada na parede, onde foi fixada a trena metálica de aço com extensão de três metros, com auxílio do esquadro de madeira. O índice de massa corporal dos pais foi calculado para a avaliação da obesidade, utilizando-se os pontos de cortes recomendados pela World Health Organization $^{17}$.

As variáveis do estudo foram obtidas com o uso de questionário pré-codificado, constituído de questões relativas às características socioeconômicas, da família, do domicílio, maternas, atividade física, características das crianças e morbidade. As variáveis maternas foram obtidas das mães biológicas, enquanto que as medidas antropométricas foram aferidas dos pais biológicos e dos pais não naturais.

As medidas antropométricas e as entrevistas foram efetuadas por alunas do curso de nutrição devidamente treinadas ${ }^{18}$. O levantamento antropométrico dos escolares foi realizado em novembro de 1999, enquanto que as entrevistas e a avaliação antropométrica com os pais aconteceram em 2000.

As variáveis contínuas foram categorizadas com base, sempre que possível, em critérios de relevância biológica das categorias ou com base 
em quartis da distribuição. A codificação das categorias das variáveis discretas não binárias foi realizada de acordo com o critério biológico, gradiente de risco observado para sobrepeso e, quando possível, as categorias foram reagrupadas conforme a similaridade de risco.

Nas análises univariadas foram estimados os valores de odds ratio e intervalo de confiança de $95 \%$, sendo que as variáveis que apresentaram $p<0,15$ foram consideradas para inclusão no modelo de regressão logística múltipla. Mas, com base em testes de distribuição de qui-quadrado e de correlação, identificaram-se e excluíram-se, em cada nível do modelo (Quadro 1), variáveis que apresentaram forte correlação com outra variável explanatória, sendo o critério de exclusão a presença de coeficiente de correlação $r \geq 0,60$. A regressão logística múltipla, não condicional, hierarquizada, foi feita usando a técnica de regressão sequencial (stepwise, backward). A partir do modelo com todas as variáveis de cada nível, excluiram-se as variáveis com menor significância (maior valor de p), sendo o modelo reestimado após cada exclusão e, assim sucessivamente, até que todas as variáveis, que permanecessem no modelo, atingissem o critério de $p<0,05$. No modelo final, as variáveis, que apresentaram nível de significância menor que 5\% foram consideradas associadas ao sobrepeso.

Os dados foram digitados em um banco no programa Epi Info versão 6.04b ${ }^{19}$. As análises univariadas e de regressão logística foram realizadas nos programas Epi Info e Stata ${ }^{20}$. O projeto de pesquisa foi aprovado pelo Comitê de Ética do Hospital Universitário Júlio Müller da Universidade Federal de Mato Grosso, credenciado pela Comissão Nacional de Ética em Pesquisa do Ministério da Saúde do Brasil.

\section{RE S U LT A D O S}

A amostra prevista para o inquérito antopométrico foi de 1300 escolares e o número de alunos que frequentavam as turmas selecionadas foi de 1329 . Destes, foram exami- nados 1256 escolares, significando uma perda de $5,5 \%$. Nesse grupo, detectou-se uma prevalência de sobrepeso (IMC $\geq P 85$ ) de $14,4 \%$, sendo de $10,5 \%$ nos meninos e de $18,7 \%$ nas meninas. Dos 1256 escolares, 1100 (87,6\%) foram entrevistados. As perdas (12,4\%) ocorreram por mudança ou erro de endereço $(n=67) \quad(n=78)$, respectivamente, recusa $(n=7)$ e entrevistas incompletas $(n=4)$. Em 1100 escolares entrevistados, 158 apresentaram sobrepeso $(14,4 \%)$ e compuseram o grupo dos casos. Dentre os demais escolares, 316 foram sorteados e incluídos no grupo controle.

Neste estudo as entrevistas foram realizadas com as mães das 474 crianças, com duração média de 26,5 minutos ( $D P \pm 8,52$ ), sendo $73,0 \%$ aplicadas nos domicílios e as demais nas escolas. O percentual de informação ignorada foi mínimo ou mesmo inexistente para a maioria das variáveis analisadas. Algumas variáveis apresentaram valores importantes de informação ignorada, como escolaridade do pai (15,8\%), posição na ocupação do chefe de família $(15,8 \%)$ e o IMC do mesmo $(28,3 \%)$.

Com relação aos 474 escolares avaliados no presente estudo, verificou-se que $50,2 \%$ eram do sexo masculino e $49,8 \%$ do sexo feminino, sendo 54,4\% com 7 anos, 20,3\% com 8 anos e apenas 3,8\% com idade de 10 ou 11 anos. Dentre os 158 escolares, que estavam com IMC $\geq 85$ percentil, 46,2\% (73) eram obesos (IMC $\geq 95$ percentil).

O grupo de criancas, casos e controles, apresentou algumas características que se destacam nas proporções exibidas. A freqüência de crianças com sobrepeso (casos), cujas mães tinham $2^{\circ}$ grau, foi maior $(44,8 \%$ ) que no grupo de crianças controle (21,3\%). A proporção de crianças no estrato de renda familiar maior que três salários mínimos foi de $26,7 \%$ nos casos e de $7,9 \%$ nos controles. A distribuição das crianças, segundo a variável número de pessoas que residem no domicílio, indicou no estrato de $\leq 4$ pessoas, prevalências de $50,0 \%$ para os casos e $34,4 \%$ para os controles. A presença de nove ou mais equipamentos domésticos no domicílio foi mais freqüente nos casos $(42,0 \%)$ que nos controles $(21,5 \%)$. 
As análises univariadas foram realizadas de acordo com os seguintes tópicos: variáveis indicadoras do nível socioeconômico, da família, domicílio, variáveis maternas, atividade física, índice de massa corporal dos pais e da criança. Encontram-se nas tabelas de 1 a 4 as variáveis que apresentaram associação estatisticamente significativa $(p<0,05)$ com o sobrepeso.

Mostraram-se associadas ao sobrepeso o nível de escolaridade da mãe e do pai, renda familiar per capita, variáveis relacionadas à ocupação da mãe e do pai, carga horária semanal de trabalho da mãe, posição na ocupação e desemprego paterno (Tabela 1). Os escolares de mães e pais com maior nível de escolaridade tiveram chances de 3,3 e 2,2 vezes, respectivamente, maior de terem sobrepeso em relação aos de baixa escolaridade. Observou-se uma relação diretamente proporcional entre a renda familiar per capita e a chance de sobrepeso.

As variáveis relativas à família e domicílio associadas ao sobrepeso foram chefe de família, número de pessoas e irmãos que residem no domicílio, presença de criança menor de cinco anos, tipo de casa, número de cômodos e número de equipamentos domésticos. A chance de sobrepeso foi maior nos filhos únicos ou que tinham apenas um irmão. Os escolares residentes em domicílios com nove ou mais equipamentos domésticos tiveram chance 3,2 vezes maior de serem obesos (Tabela 2).

A Tabela 3 apresenta os resultados da análise referente às variáveis maternas e da criança, como o número de uniões, separação após o nascimento da criança, intervalo interpartal,

Tabela 1. Número de casos, controles, odds ratio e IC (95\%), segundo variáveis socioeconômicas. Cuiabá, 2000.

\begin{tabular}{|c|c|c|c|c|c|}
\hline Variável & Caso & Controle & OR bruta & IC $95 \%$ & $p\left(\chi^{2}\right)$ \\
\hline Escolaridade da mãe & & & & & $<0,001$ \\
\hline Até $1^{\circ}$ grau incompleto & 62 & 197 & 1 & - & \\
\hline $1^{\circ}$ grau completo & 23 & 47 & 1,55 & $0,87-2,76$ & \\
\hline $2^{\circ}$ grau completo e superior & 69 & 66 & 3,32 & $2,13-5,17$ & \\
\hline Escolaridade do pai & & & & & 0,004 \\
\hline $1^{\circ}$ grau incompleto & 60 & 156 & 1 & - & \\
\hline $1^{\circ}$ grau completo & 20 & 41 & 1,27 & $0,69-2,34$ & \\
\hline $2^{\circ}$ grau completo e superior & 56 & 66 & 2,21 & $1,39-3,51$ & \\
\hline Renda familiar per capita & & & & & $<0,001$ \\
\hline$\leq 0,5$ & 38 & 130 & 1 & - & \\
\hline $0,51-2,0$ & 65 & 147 & 1,51 & $0,95-2,41$ & \\
\hline $2,01-3,0$ & 12 & 13 & 3,16 & $1,33-7,49$ & \\
\hline 3,01 e mais & 42 & 25 & 5,75 & $3,11-10,61$ & \\
\hline Carga horária semanal da mãe & & & & & 0,016 \\
\hline Não trabalham & 49 & 135 & 1 & - & \\
\hline$\leq 20$ & 17 & 45 & 1,04 & $0,55-1,99$ & \\
\hline $21-39$ & 31 & 53 & 1,61 & $0,93-2,79$ & \\
\hline$\geq 40$ & 56 & 75 & 2,06 & $1,28-3,31$ & \\
\hline $\begin{array}{l}\text { Posição na ocupação/chefe não } \\
\text { trabalham e conta própria }\end{array}$ & & & & & $<0,001$ \\
\hline Não estabelecido & 35 & 92 & 1 & - & \\
\hline Assalariado & 67 & 142 & 1,24 & $0,76-2,02$ & \\
\hline Conta própria estabelecido & 20 & 26 & 2,02 & $1,00-4,07$ & \\
\hline Empregador & 14 & 3 & 12,27 & $3,32-45,29$ & \\
\hline Desemprego & & & & & 0,006 \\
\hline Sim & 56 & 153 & 1 & - & \\
\hline Não & 97 & 153 & 1,73 & $1,16-2,58$ & \\
\hline
\end{tabular}


10 | L.V. GUIMARÃES et al.

Tabela 2. Número de casos, controles, odds ratio e IC (95\%), segundo variáveis relativas à família e domicílio. Cuiabá, 2000.

\begin{tabular}{|c|c|c|c|c|c|}
\hline Variável & Caso & Controle & OR bruta & IC $95 \%$ & $p\left(\chi^{2}\right)$ \\
\hline Chefe de família & & & & & 0,002 \\
\hline Outra pessoa & 10 & 39 & 1,00 & & \\
\hline Pai ou mãe & 122 & 254 & 1,87 & $0,90-3,88$ & \\
\hline Pai e mãe & 26 & 23 & 4,41 & $1,80-10,77$ & \\
\hline Número de pessoas & & & & & $<0,001$ \\
\hline 6 e mais & 34 & 123 & 1,00 & - & \\
\hline 5 & 45 & 84 & 1,94 & $1,15-3,27$ & \\
\hline$\leq 4$ & 79 & 109 & 2,62 & $1,62-4,23$ & \\
\hline Número de irmãos & & & & & $<0,001$ \\
\hline 3 e mais & 20 & 88 & 1,00 & & \\
\hline 2 & 45 & 99 & 2,00 & $1,10-3,64$ & \\
\hline$\leq 1$ & 93 & 129 & 3,17 & $1,82-5,52$ & \\
\hline Crianças com $<5$ anos & & & & & 0,024 \\
\hline Sim & 39 & 110 & 1,00 & & \\
\hline Não & 119 & 206 & 1,63 & $1,06-2,50$ & \\
\hline Tipo de casa & & & & & $<0,001$ \\
\hline Acabamento incompleto e outro & 69 & 213 & 1,00 & & \\
\hline Alvenaria acabamento completo & 89 & 103 & 2,67 & $1,80-3,95$ & \\
\hline Número de cômodos & & & & & $<0,001$ \\
\hline$\leq 3$ & 20 & 74 & 1,00 & & \\
\hline $4-5$ & 58 & 135 & 1,59 & $0,88-2,84$ & \\
\hline $5-7$ & 38 & 67 & 2,10 & $1,11-3,96$ & \\
\hline$\geq 8 \mathrm{~s}$ & 42 & 40 & 3,90 & $2,01-7,49$ & \\
\hline Número de equipamento doméstico & & & & & $<0,001$ \\
\hline$\leq 6$ & 56 & 183 & 1,00 & & \\
\hline $7-8$ & 35 & 65 & 1,76 & $1,06-2,92$ & \\
\hline$\geq 9$ & 67 & 68 & 3,22 & $2,05-5,05$ & \\
\hline
\end{tabular}

idade materna no nascimento da criança, peso e comprimento ao nascer, sexo e idade. Escolares cujas mães tinham vivenciado uma união conjugal e, que na época do nascimento da criança, estavam com idade entre 25 e 29 anos, apresentaram maior chance de sobrepeso. $O$ peso ao nascer, sexo e idade foram associados com sobrepeso. Os escolares que tiveram maior chance $(\mathrm{OR}=2,15)$ de sobrepeso foram os de menor idade (6 a 7 anos).

As variáveis relativas à atividade física do escolar que se mostraram associadas com o sobrepeso foram: prática de educação física e esporte, horas de brincadeira de rua na semana, horas de sono por dia e meio de locomoção utilizado para ir à escola. O índice de massa corporal dos pais mostrou forte associação com o sobrepeso nos escolares (Tabela 4).

Entre as variáveis socioeconômicas, incluídas no nível 1 do modelo da análise de regressão logística múltipla hierarquizada, as que permaneceram com significância estatística foram a escolaridade materna e a renda familiar per capita (Tabela 5).

O nível 2 refere-se à análise das variáveis relativas à família, ao domicílio e maternas (Quadro 1). Nesta etapa foram mantidas as variáveis escolaridade materna e renda familiar per capita e, as variáveis do nível 2 que permaneceram no modelo foram o número de uniões desde o nascimento da criança, idade materna no nascimento da criança e número de irmãos. 
Tabela 3. Número de casos, controles, odds ratio e IC (95\%), segundo variáveis maternas e da criança. Cuiabá, 2000.

\begin{tabular}{|c|c|c|c|c|c|}
\hline Variável & Caso & Controle & OR bruta & IC $95 \%$ & $p\left(\chi^{2}\right)$ \\
\hline Número de uniões & & & & & 0,006 \\
\hline$\geq 2$ & 8 & 42 & 1,00 & & \\
\hline Nenhuma & 10 & 24 & 2,19 & $0,76-6,30$ & \\
\hline 1 & 130 & 218 & 3,13 & $1,42-6,87$ & \\
\hline Separação conjugal & & & & & 0,012 \\
\hline $\operatorname{sim}$ & 33 & 96 & 1,00 & & \\
\hline Não & 115 & 188 & 1,77 & $1,12-2,81$ & \\
\hline Intervalo Interpartal (meses) & & & & & 0,003 \\
\hline$\leq 35$ & 34 & 111 & 1,00 & & \\
\hline$>35$ & 58 & 83 & 2,28 & $1,36-3,80$ & \\
\hline Filho único & 56 & 90 & 2,03 & $1,22-3,36$ & \\
\hline Idade Materna (anos) & & & & & 0,009 \\
\hline$\leq 24$ & 60 & 158 & 1,00 & & \\
\hline $25-29$ & 50 & 67 & 1,96 & $1,22-3,15$ & \\
\hline$\geq 30$ & 38 & 58 & 172 & $1,04-2,86$ & \\
\hline Sexo & & & & & 0,001 \\
\hline Masculino & 63 & 175 & 1,00 & & \\
\hline Feminino & 95 & 141 & 1,87 & $1,27-2,76$ & \\
\hline Idade (em anos) & & & & & 0,001 \\
\hline$\geq 8$ & 30 & 106 & 1,00 & & \\
\hline $6-7$ & 128 & 210 & 2,15 & $1,36-3,41$ & \\
\hline Peso ao nascer (g) & & & & & 0,006 \\
\hline$\leq 3500$ & 97 & 216 & 1,00 & & \\
\hline$>3500$ & 53 & 64 & 1,84 & $1,19-2,85$ & \\
\hline
\end{tabular}

Quadro 1. Hierarquização do modelo de regressão multivariada utilizado para a análise de sobrepeso em escolares. Cuiabá, 2000.

\begin{tabular}{|c|c|c|}
\hline Nível & Características & Variáveis \\
\hline $1^{\circ}$ & 1.1. Socioeconômica & $\begin{array}{l}\text { Nível de escolaridade dos pais, renda familiar, carga horária de trabalho e número de } \\
\text { empregos pregressos da mãe, condição de ocupação do pai/chefe de família, posição } \\
\text { e tempo de ocupação do pai, número de empregos pregressos e desemprego do pai. }\end{array}$ \\
\hline \multirow[t]{3}{*}{$2^{\circ}$} & 2.1. Família & $\begin{array}{l}\text { Situação conjugal da mãe no nascimento da criança e atual, número de uniões e } \\
\text { separações da mãe desde o nascimento da criança, presença dos pais biológicos na } \\
\text { casa, chefe de família, número de pessoas, irmãos, crianças < } 5 \text { anos, número de } \\
\text { pessoas por quarto. }\end{array}$ \\
\hline & 2.2. Materna & Intervalo interpartal, idade materna e ordem de nascimento. \\
\hline & 2.3. Domicílio & $\begin{array}{l}\text { Tipo e condição de ocupação da casa, tipo de abastecimento de água, esgoto sanitário, } \\
\text { destino do lixo e número de equipamentos domésticos. }\end{array}$ \\
\hline \multirow[t]{3}{*}{$3^{\circ}$} & 3.1. Atividade física & $\begin{array}{l}\text { Prática de esporte, tempo de educação física, tempo que anda de bicicleta, tempo de } \\
\text { brincadeiras, locomoção para escola, horas de sono e assistindo TV. }\end{array}$ \\
\hline & 3.2. Morbidade & Presença de morbidade e internação hospitalar. \\
\hline & 3.3. IMC dos pais & IMC da mãe e IMC do pai. \\
\hline $4^{\circ}$ & 4.1. Relativas à criança & Sexo, idade, peso e comprimento ao nascer e amamentação. \\
\hline
\end{tabular}

IMC= índice de massa corporal. 
Tabela 4. Número de casos, controles, odds ratio e IC (95\%), segundo variáveis relativas à atividade física e IMC dos pais. Cuiabá, 2000 .

\begin{tabular}{|c|c|c|c|c|c|}
\hline Variável & Caso & Controle & OR bruta & IC 95\% & $p\left(\chi^{2}\right)$ \\
\hline Educação física & & & & & 0,017 \\
\hline Não fazem & 29 & 79 & 1 & & \\
\hline$<90$ minutos & 29 & 31 & 2,55 & $1,25-5,22$ & \\
\hline$\geq 90$ minutos & 100 & 206 & 1,32 & $0,79-2,22$ & \\
\hline Prática de esporte & & & & & 0,008 \\
\hline Não & 134 & 293 & 1 & & \\
\hline $\operatorname{sim}$ & 24 & 23 & 2,28 & $1,24-4,19$ & \\
\hline Horas de brincadeiras/semana & & & & & 0,009 \\
\hline$\geq 26,01$ & 18 & 62 & 1 & & \\
\hline $26,00-10,01$ & 89 & 186 & 1,65 & $0,92-2,95$ & \\
\hline$\leq 10,00$ & 51 & 68 & 2,58 & $1,36-4,89$ & \\
\hline Horas de sono/dia & & & & & 0,001 \\
\hline$>10$ & 42 & 121 & 1 & & \\
\hline $10-8,01$ & 85 & 167 & 1,47 & $0,93-2,33$ & \\
\hline$\leq 8$ & 31 & 28 & 3,19 & $1,64-6,22$ & \\
\hline Meio de locomoção utilizado para ir à escola & & & & & $<0,001$ \\
\hline A pé & 104 & 259 & 1 & & \\
\hline Transporte coletivo e outro & 19 & 27 & 1,75 & $0,93-3,29$ & \\
\hline Carro próprio & 33 & 26 & 3,16 & $1,80-5,54$ & \\
\hline IMC da mãe & & & & & $<0,001$ \\
\hline$\leq 24,99$ & 53 & 170 & 1 & & \\
\hline $25,00-39,99$ & 43 & 95 & 1,45 & $0,90-2,33$ & \\
\hline$\geq 30$ & 55 & 40 & 4,41 & $2,65-7,35$ & \\
\hline IMC do pai & & & & & $<0,001$ \\
\hline$\leq 24,99$ & 34 & 110 & 1 & & \\
\hline $25-29,99$ & 55 & 85 & 2,09 & $1,25-3,50$ & \\
\hline$\geq 30$ & 28 & 28 & 3,24 & $1,69-6,20$ & \\
\hline
\end{tabular}

IMC = índice de massa corporal.

Do nível hierárquico 3, as variáveis mantidas no modelo foram IMC da mãe e do pai, horas de brincadeira de rua e horas de sono. A variável tempo de brincadeira de rua, na categoria de $<10$ horas, apresentou-se com uma $\mathrm{OR}_{\text {ajustada }}$ de 2,58 (IC95\% 1,00-6,63) ficando no limiar de significância estatística. A variável horas de

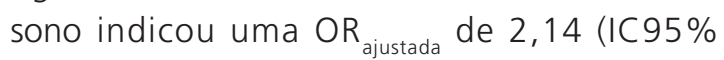
$0,96-4,78)$ para os escolares que dormiam 8 horas ou menos por dia, comparados aos que dormiam mais de 8 horas, que ficou ligeiramente acima do limiar de significância estatística. Ambas foram mantidas no modelo final desse nível hierárquico.

No nível 4 fez-se a regressão logística, inserindo na análise as variáveis peso e comprimento ao nascer, aleitamento materno, idade e sexo. Permaneceram com significância estatística as variáveis sexo e peso ao nascer. Não foi verificada interação estatisticamente significante entre as variáveis que persistiram no modelo de regressão múltipla.

Em síntese, a análise de regressão logística múltipla hierarquizada identificou o seguinte conjunto de variáveis como associadas ao sobrepeso: a escolaridade materna e a renda familiar per capita (nível 1); o número de uniões da mãe desde o nascimento da criança, o número de irmãos e a idade materna no nascimento da criança (nível 2); as horas de brincadeiras por semana, o IMC da mãe e do pai (nível 3), o sexo e o peso ao nascer (nível 4). 
Tabela 5. Resultado da análise de regressão logística múltipla hierarquizada. Cuiabá, 2000.

\begin{tabular}{|c|c|c|c|c|c|}
\hline Modelo & $\mathrm{OR}_{\text {bruta }}$ & $\mathrm{OR}_{\text {ajustada }}$ & IC 95\% & $p$ categoria & $p$ variável \\
\hline \multicolumn{6}{|c|}{ - Características socioeconômicas } \\
\hline Escolaridade da mãe & & & & & 0,056 \\
\hline Até $1^{\circ}$ grau incompleto & 1,00 & 1,00 & & & \\
\hline $1^{\circ}$ grau completo & 1,55 & 1,38 & {$[0,76-2,49]$} & 0,278 & \\
\hline $2^{\circ}$ grau e superior & 3,32 & 1,91 & {$[1,12-3,26]$} & 0,016 & \\
\hline Renda familiar per capita & & & & & 0,003 \\
\hline$\leq 0,50$ & 1,00 & 1,00 & & & \\
\hline $0,51-2,00$ & 1,51 & 1,31 & {$[0,81-2,14]$} & 0,26 & \\
\hline $2,01-3,00$ & 3,16 & 2,18 & {$[0,87-5,46]$} & 0,097 & \\
\hline$\geq 3,01$ & 5,75 & 3,75 & {$[1,82-7,71]$} & 0 & \\
\hline \multicolumn{6}{|c|}{ - Características da família e maternas } \\
\hline Número de irmãos & & & & & 0,126 \\
\hline$\geq 3$ & 1,00 & 1,00 & {$[0,78-2,99]$} & 0,211 & \\
\hline 2 & 2,00 & 1,53 & {$[1,01-3,72]$} & 0,046 & \\
\hline$\leq 1$ & 3,17 & 1,94 & & & \\
\hline Número de uniões da mãe & & & & & 0,052 \\
\hline$\geq 2$ & 1,00 & 1,00 & {$[0,62-5,84]$} & 0,254 & \\
\hline Nenhuma & 2,19 & 1,91 & {$[1,12-5,73]$} & 0,026 & \\
\hline 1 & 3,13 & 2,53 & & & \\
\hline Idade materna & & & & & 0,062 \\
\hline$\leq 24$ & 1,00 & 1,00 & {$[1,04-2,90]$} & 0,032 & \\
\hline $25-29$ & 1,96 & 1,74 & {$[0,92-2,80]$} & 0,098 & \\
\hline$\geq 30$ & 1,72 & 1,6 & & & \\
\hline \multicolumn{6}{|l|}{ - Atividade física } \\
\hline Tempo que brinca/semana & & & & & 0,129 \\
\hline 26,01 & 1,00 & 1,00 & {$[0,83-4,78]$} & 0,121 & \\
\hline $10,01-26$ & 1,65 & 1,99 & {$[1,00-6,63]$} & 0,049 & \\
\hline $0-10$ & 2,58 & 2,58 & & & \\
\hline Horas de sono/dia & & & & & 0,062 \\
\hline$\geq 8,01$ & 1,00 & 1,00 & {$[0,96-4,78]$} & 0,062 & \\
\hline$\leq 8$ & 2,51 & 2,14 & & & \\
\hline \multicolumn{6}{|l|}{ - Antropométricas } \\
\hline IMC da mãe & & & & & $<0,001$ \\
\hline \multicolumn{6}{|l|}{$\leq 24$} \\
\hline $25-29$ & 1,45 & 1,68 & {$[0,89-3,16]$} & 0,107 & \\
\hline$\geq 30$ & 4,41 & 7,27 & {$[3,36-15,75]$} & $<0,001$ & \\
\hline IMC do pai & & & & & 0,053 \\
\hline \multicolumn{6}{|l|}{$\leq 24$} \\
\hline $25-29$ & 2,09 & 1,48 & {$[0,78-2,82]$} & 0,229 & \\
\hline$\geq 30$ & 3,24 & 2,65 & {$[1,20-5,85]$} & 0,015 & \\
\hline Sexo & & & & & 0,009 \\
\hline Masculino & 1,00 & 1,00 & & & \\
\hline Feminino & 1,87 & 2,15 & {$[1,20-3,86]$} & 0,010 & \\
\hline Peso ao nascer & & & & & 0,015 \\
\hline$\leq 3500$ & 1,00 & 1,00 & & & \\
\hline$>3500$ & 1,84 & 2,27 & {$[1,18-4,39]$} & 0,015 & \\
\hline
\end{tabular}

IMC = índice de massa corporal. 


\section{I S C US S Ã O}

Os resultados neste estudo revelam que, nos escolares de Cuiabá, a obesidade é mais freqüente nos segmentos populacionais de melhor nível socioeconômico, associação verificada de forma consistente tanto com a variável renda quanto com a variável escolaridade materna.

Verificou-se que o alto nível de escolaridade materna correspondeu a maior chance de sobrepeso dos escolares. A OR $_{\text {ajustada }}$ de sobrepeso foi de 1,9 nos escolares cujas mães possuíam maior nível de escolaridade em relação àquelas com primeiro grau incompleto. Estudo, realizado por Ribeiro et al. ${ }^{5}$, encontrou uma OR de 1,85 (IC 1,25-4,37) de obesidade em escolares de 7 a 10 anos, em São Paulo, cujas mães tinham mais de quatro anos de escolaridade, comparados com os filhos de mães com escolaridade mais baixa.

A razão de chances foi de 3,75 para os escolares com maior renda familiar per capita em relação aos de menor renda; esta razão é semelhante à de 3,8, observada por Engestron \& Anjos ${ }^{7}$, em crianças brasileiras menores de 10 anos, cujas famílias possuíam maior renda familiar em relação às de baixa renda. Investigação realizada com escolares da Tailândia, com idade entre 6 a 13 anos, observou um aumento do risco de obesidade com o aumento da renda familiar ${ }^{8}$. Também em pré-escolares da rede pública, de cidade do interior de São Paulo, foi observada maior prevalência de obesidade no grupo de crianças de nível socioeconômico mais alto²1.

Entretanto, estudos realizados em países mais desenvolvidos têm apontado maior prevalência de obesidade nos segmentos populacionais de pior nível socioeconômico. Em estudo sobre percepção materna e sobrepeso de pré-escolares norte-americanos, Baughcum et al. ${ }^{9}$ relatam que a freqüência de obesidade foi maior entre filhos de mães com baixa escolaridade e verificaram que essas possuíam dificuldade para perceber o excesso de peso dos seus filhos, quando comparadas com as mães de nível de escolaridade mais alto.
É freqüente, nas áreas menos desenvolvidas, as populações pobres serem menos vulneráveis à obesidade, cuja prevalência tende a aumentar com o nível socioeconômico ${ }^{22}$. Nestas regiões, a percepção da mãe quanto à saúde nutricional da criança poderia estar mais relacionada com a quantidade do consumo alimentar do que com as qualidades nutritivas da alimentação, mesmo nos segmentos de melhor nível socioeconômico. A adoção de padrão alimentar com dieta rica em gordura saturada e açúcar e com baixo teor de fibras e a redução dos níveis de atividade física têm sido consideradas as responsáveis pelo elevado nível de sobrepeso ${ }^{23}$.

Mas, a maior presença da obesidade nos níveis socioeconômicos mais elevados, nos países de menor desenvolvimento, parece estar em processo de mudança. Estudo brasileiro observou em sua população adulta feminina, em 1997, maior prevalência de obesidade no quartil de mais baixa renda, invertendo o padrão que havia sido observado em 1989 e $1975^{24}$.

A variável número de uniões mostrou-se associada ao sobrepeso, com $\mathrm{OR}_{\text {ajustada }}$ de 2,53 para as crianças cujas mães haviam tido apenas uma união conjugal em relação às que haviam tido duas ou mais. Este achado indica que, mesmo quando controlado o efeito de variáveis socioeconômicas e das demais variáveis do modelo, a estabilidade da situação conjugal parece exercer influência sobre o sobrepeso dos escolares na população estudada. Investigação realizada em escolares, por Wolfe et al. ${ }^{25}$, observou interação entre nível socioeconômico e composição familiar, indicando que, nas crianças com pais presentes no domicílio, o sobrepeso era maior nas de nível socioeconômico mais baixo, ocorrendo o inverso com crianças de pais separados.

Quanto à idade materna, observou-se uma $\mathrm{OR}_{\text {ajustada }}$ de 1,74 nos filhos de mães de 25 a 29 anos em relação aos filhos de mães mais jovens. Também em estudo realizado com 2379 meninas norte-americanas, entre 9 e 10 anos, foi encontrado aumento da prevalência de obesidade com o aumento da idade materna, com razão de 
chances de 1,14 para cada aumento de 5 anos na idade materna ${ }^{26}$. É possível que, na população estudada, as mães com maior idade tendam a proteger mais os filhos, o que pode incluir maior oferta de alimentos.

A variável número de irmãos manteve-se associada ao sobrepeso na análise de regressão múltipla, com $\mathrm{OR}_{\text {ajustada }}$ de 1,94 para os escolares com até um irmão, comparados com aqueles que tinham três irmãos ou mais. A superproteção materna e a maior oferta de alimentos têm sido aventadas como possíveis mecanismos de explicação para a maior prevalência de obesidade nos filhos únicos ${ }^{8}$. Mas, os autores apontam também que a atividade física e as brincadeiras seriam mais freqüentes em famílias com maior número de crianças ${ }^{8,10}$.

O número de horas semanais em que a criança participa de brincadeiras de rua mostrou tendência de associação dose-resposta, apresentando diminuição do sobrepeso à medida que aumentava o tempo de brincadeiras. $\mathrm{A} \mathrm{OR}_{\text {ajustada }}$ na categoria de 10 horas ou menos foi de 2,58, comparada aos escolares que brincavam mais de 26 horas por semana. Guillaume et al. ${ }^{27}$ têm verificado essa associação. A relação entre atividade física, obesidade e outros fatores de risco para doenças cardiovasculares foi analisada em crianças de uma província da Bélgica. A atividade física foi medida pela participação em práticas esportivas e a inatividade física estimada pelo tempo destinado a assistir televisão. Os autores concluíram que $12 \%$ da obesidade poderia ser atribuível à baixa intensidade de atividade física.

A variável tempo de sono diário indicou uma chance maior de sobrepeso nos escolares que dormiam no máximo oito horas por dia, porém o intervalo de confiança incluiu a unidade. Sekine et al. ${ }^{28,29}$ constataram uma relação dose-resposta entre horas de sono e obesidade. $\mathrm{AOR}_{\text {ajustada }}$ foi de 2,87 (IC95\% 1,61-5,05) para aquelas crianças que dormiam menos de 8 horas, em comparação com as crianças que dormiam mais de 10 horas $^{29}$. Giugliano \& Carneiro 6 observaram em escolares uma correlação inversa e significativa $(r=-0,278$; $p<0,02)$ entre o percentual de gordura corporal e o tempo diário médio de sono. A relação entre horas de sono e sobrepeso tem sido evidenciada na literatura, mas a explicação da associação ainda não está elucidada.

Foi observada associação estatística forte entre IMC dos pais, especialmente o da mãe, e o sobrepeso dos escolares. As $\mathrm{OR}_{\text {ajustadas }}$ foram, respectivamente, de 7,27 e de 2,65 para os escolares cujas mães e pais possuíam IMC igual ou superior a 30 , quando comparados com aqueles cujos pais tinham o IMC inferior a 25.

Em estudo realizado com uma coorte de crianças de 0 a 8 anos de idade, acompanhadas durante seis anos, observou-se razão de incidência acumulada ajustada de obesidade de 3,69 para as crianças cujas mães estavam com IMC maior ou igual a 30, tendo por referência as crianças de mães com IMC entre 20 e $25^{3}$. Ribeiro et al. ${ }^{5}$ verificaram que a chance dos escolares serem obesos foi de 2,5 (IC 95\% 1,43-4,37) vezes maior quando o IMC dos pais era maior que 30 .

A associação da obesidade infantil com valores mais altos do IMC dos pais pode ser decorrente de herança genética e/ou de fatores relacionados às condições de vida, que atuariam independentemente ou facilitando a expressão genética ${ }^{8}$. A capacidade de consumo e o estilo de vida, incluindo o padrão alimentar e o de atividade física, podem parcialmente explicar a presença concomitante de obesidade em pais e filhos ${ }^{8}$.

O peso ao nascer manteve-se associado com o sobrepeso, após o ajuste por potenciais variáveis de confusão, com uma razão de chances de 2,27 para as crianças que nasceram com peso superior a 3500 gramas, comparadas com aquelas que nasceram com peso inferior ou igual a 3500 gramas. Os resultados encontrados na literatura ainda são contraditórios sobre a influência do peso ao nascer na obesidade. Estudo realizado com escolares em São Paulo também encontrou maior chance de obesidade $(O R=1,83)$ em crianças que tinham nascido com peso $\geq 3500$ gramas $^{5}$. Garnett et al. ${ }^{14}$, entretanto, verificaram que as crianças 
que nasceram com peso $<3.000 \mathrm{~g}$ apresentaram, aos 7 e 8 anos, maior escore de peso e de gordura abdominal.

A maior ocorrência de sobrepeso nas meninas, como observado neste estudo, concorda com resultados verificados por outros autores ${ }^{5,11}$. No estudo realizado por Crespo et al. ${ }^{10}$ foi observado que as meninas apresentavam menor grau de atividade física e maior tempo dedicado a assistir televisão que os meninos. Marins et al. ${ }^{1}$, entretanto, observaram o contrário em crianças e adolescentes do Rio de Janeiro, onde o sobrepeso é maior nos meninos.

Evidenciou-se com o presente estudo que o sobrepeso de escolares encontra-se condicionado aos padrões socioeconômicos e familiares e às características e comportamentos das crianças. $\mathrm{O}$ efeito do padrão socioeconômico se expressa nas associações observadas com as variáveis renda e escolaridade materna. A influência dos fatores familiares foi observada pelo efeito das variáveis número de uniões conjugais da mãe, idade materna e número de irmãos. Esta última variável, bem como, horas de brincadeira e de sono, referem-se à dinâmica presente na família. O sexo da criança e o peso ao nascer expressam características específicas da criança que exercem influência no padrão nutricional. Os achados relativos aos IMC dos pais, em especial, à magnitude da associação com o IMC materno, apontam o papel importante exercido pela mãe na relação criança-ambiente, que iniciada na gestação, significará compartilhar condições sócio-ambientais, modos e estilos de vida, que incluem os hábitos alimentares e de atividade física com repercussão significativa no estado nutricional. Os resultados indicam que medidas de intervenção precisam levar em conta o contexto familiar e socioeconômico da criança. Políticas de alimentação e nutrição precisariam assegurar atuações no ambiente escolar, com medidas efetivas do setor saúde para a promoção de alimentação saudável, da vigilância dos problemas nutricionais e do acompanhamento dos escolares com distúrbios já detectados.

\section{A GRADECIMENTOS}

Às diretoras das escolas pelo apoio e aos escolares pela participação neste estudo. À Fundação de Amparo à Pesquisa do Estado de Mato Grosso FAPEMAT, FUNASA - MS e Secretaria de Estado de Saúde MT pelo suporte financeiro à pesquisa de campo. À CAPES pelo auxílio financeiro recebido no doutoramento.

\section{REFERÊ NCIAS}

1. Marins VM, Almeida RMVR, Pereira RA, Barros, MBA. Overweight and risk of overweight in schoolchildren in the city of Rio de Janeiro, Brazil: prevalence and characteristics. Ann Trop Paediatrics. 2002; 22(2):137-44

2. Wang $Y$, Monteiro CA, Popkin BM. Trends of obesity and underweight in older children and adolescents in the United States, Brazil, China, and Russia. Am J Clin Nutr. 2002; 75(6):971-7.

3. Doak CM, Adair LS, Bentley M, Monteiro C, Popkin BM. The dual burden households and nutrition transition paradox. Int J Obes Relat Metab Disord. 2005; 129(1):129-36.

4. Ramos AMP, Barros Filho AA. Prevalência da obesidade em adolescentes de Bragança Paulista e sua correlação com a obesidade dos pais. Arq Bras Endocrinol Metab. 2003; 47:663-8.

5. Ribeiro IC, Taddei JAAC, Colugnatti F. Obesity among children attending elementary public schools in São Paulo, Brazil: a case-control study. Public Health Nutr. 2003; 6(7):659-63.

6. Giugliano R, Carneiro EC. Fatores associados à obesidade em escolares. J Pediatr. 2004; 80(1):17-22.

7. Engestron EM, Anjos LA. Relação entre o estado nutricional materno e sobrepeso nas crianças brasileiras. Rev Saúde Pública. 1996; 30(3):233-9.

8. Mo-Suwam L, Geater AF. Risk factors for childhood obesity in a transitional society in Thailand. Int J Obes Relat Metab Disord. 1996; 20(8):697-703.

9. Baughcum AE, Chamberlin LA, Deeks CM, Powers SW, Whitaker RC. Maternal perceptions of overweight preschool children. Pediatrics. 2000;106(6):1380-6.

10. Frenne LM, Zaragozano JF, Otero JMG, Aznar M, Sánchez B. Actividad física y ocio en jóvenes. II: Relación con los hábitos dietéticos. An Esp Pediatr. 1997; 46(2):126-32.

11. Crespo CJ, Smit E, Troiano RP, Bartlett SJ, Macera CA, Anderson RE. Television watching, energy 
intake, and obesity in US children: results from the third National Health of Nutrition Examination Survey, 1988-1994. Arch Pediatr Adolesc Med. 2001; 155(3):360-5.

12. Marins VMR, Almeida RMVR, Pereira RA, Barros MBA. The relationship between parental nutritional status and overweight children/ adolescents in Rio de Janeiro, Brazil, Public Health. 2004; 118(1):43-9.

13. Dietz WH. Critical periods in chidhood for the development of obesity. Am J Clin Nutr. 1994; 59:955-9.

14. Garnett SP, Cowell CT, Baur LA, Fay RA, Lee J, Coakely J, et al. Abdominal fat and birth size in healthy prepubertal children. Int J Obes Relat Metab Disord. 2001; 25:1667-73.

15. Schelesselman JJ, Stolley PD. Case-control studies. New York: Oxford University Press; 1982.

16. Must A, Dallal GE, Dietz WH. Reference data for obesity: $85^{\text {th }}$ and $95^{\text {th }}$ percentiles of body mass index (wt/ht ${ }^{2}$ ) and triceps skinfold thickness. Am J Clin Nutr. 1991; 53(4):839-46. Erratum in: Am J Clin Nutr. 1991; 54(5):773.

17. World Health Organization. Physical status: the use and interpretation of antropometry. Geneva: WHO; 1995. Thecnical Reports Series 854.

18. Jelliffe DB Evaluación del estado de nutrición de la comunidad. Ginebra: OMS; 1968. Séries de Monografias, 53.

19. Dean AG, Dean JA, Coulombier D; Brendel KA, Smith DC, Burton $A H$, et al. Epi Info [computer program]. Version 6: a word processing database, and statistics program for epidemiology on microcomputers. Atlanta, Georgia: Centers of Disease Control and Prevention; 1994.

20. Stata Corporation Stata Statistical [computer program]. Release 5.0 reference p-z. Texas: College Station; 1997.
21. Guimarães LV, Barros MB. A diferenciação do estado nutricional em pré-escolares de rede pública e a transição nutricional. J Pediatr. 2001; 77(5): 381-6.

22. Monteiro CA, Benício MH D’A, Conde WL, Popkin BM. Shifting obesity trends in Brazil. Eur J Clin Nut. 2000; 54(4):342-6.

23. Popkin BM. Nutritional patterns and transitions. Pop Dev. 1993; 19:138-57.

24. Monteiro CA, Conde WL. A tendência secular da desnutrição e da obesidade na infância na cidade de São Paulo (1974-1996). Rev Saúde Pública. 2000; 34(Supl 6):52-61.

25. Wolfe WS, Campbell CC, Frongillo, EA, Haas JD, Thomas AM. Overweight schoolchildren in New York State: Prevalence and characteristics. Am J Public Health. 1994; 84(5):807-13.

26. Patterson ML, Stern S, Crawford PB, McMahon RP, Similo SL, Schreiber GB, et al. Sociodemographic factors and obesity in preadolescent black and white girls: NHLBI's Growth and Health Study. J Natl Med Assoc. 1997; 89(9):594-600.

27. Guillaume M, Lapidus L, Bjorntorp P, Lambert A. Physical activity, obesity and cardiovascular risk factors in children. The Belgian Luxembourg Child Study II. Obes Res. 1997; 5(6):549-56.

28. Sekine M, Yamagami T, Hamanishi S, Handa K, Saito T, Nanri S, et al. Parental obesity, lifestyle factors and obesity in preschool children: results of the Toyama Birth Cohort Study. J Epidemiol. 2002; 12(1):33-9.

29. Sekine M, Yamagami T, Handa K, Saito T, Nanri S, Kawaminami K, et al. A dose-response betwen short sleeping hours and obesity in childhood: results of the Toyama Birth Cohort Study. Child Care Health Dev. 2002; 28(2):163-70.

Recebido em: 25/10/2004

Versão final reapresentada em: 23/5/2005 Aprovado em: 29/6/2005 